\title{
The role of gene variants in the pathogenesis of neurodegenerative disorders as revealed by next generation sequencing studies: a review
}

\author{
Shirley Yin-Yu Pang ${ }^{1}$, Kay-Cheong Teo ${ }^{1}$, Jacob Shujui Hsu², Richard Shek-Kwan Chang ${ }^{1}$, Miaoxin Li ${ }^{2,3,4}$, \\ Pak-Chung Sham ${ }^{2}$ and Shu-Leong Ho ${ }^{1 *}$
}

\begin{abstract}
The clinical diagnosis of neurodegenerative disorders based on phenotype is difficult in heterogeneous conditions with overlapping symptoms. It does not take into account the disease etiology or the highly variable clinical course even amongst patients diagnosed with the same disorder. The advent of next generation sequencing (NGS) has allowed for a system-wide, unbiased approach to identify all gene variants in the genome simultaneously. With the plethora of new genes being identified, genetic rather than phenotype-based classification of Mendelian diseases such as spinocerebellar ataxia (SCA), hereditary spastic paraplegia (HSP) and Charcot-Marie-Tooth disease (CMT) has become widely accepted. It has also become clear that gene variants play a role in common and predominantly sporadic neurodegenerative diseases such as Parkinson's disease (PD) and amyotrophic lateral sclerosis (ALS). The observation of pleiotropy has emerged, with mutations in the same gene giving rise to diverse phenotypes, which further increases the complexity of phenotype-genotype correlation. Possible mechanisms of pleiotropy include different downstream effects of different mutations in the same gene, presence of modifier genes, and oligogenic inheritance. Future directions include development of bioinformatics tools and establishment of more extensive public genotype/phenotype databases to better distinguish deleterious gene variants from benign polymorphisms, translation of genetic findings into pathogenic mechanisms through in-vitro and in-vivo studies, and ultimately finding disease-modifying therapies for neurodegenerative disorders.
\end{abstract}

Keywords: Next generation sequencing, Neurodegenerative diseases, Genetics, Pleiotropy

\section{Background}

Traditionally, neurological disorders have been classified and diagnosed based on clinical features such as symptom-onset and disease course, and characterization of physical signs to allow localization of abnormalities in the nervous system. While most acute neurological conditions can be diagnosed with reasonable certainty, the same cannot be said to be true in chronic neurodegenerative conditions, where the definitive diagnosis can often only be ascertained by specific pathologic findings. For example, studies have shown that the accuracy of

\footnotetext{
* Correspondence: slho@hku.hk

${ }^{1}$ Division of Neurology, Department of Medicine, Queen Mary Hospital,

University of Hong Kong, Hong Kong, People's Republic of China

Full list of author information is available at the end of the article
}

Parkinson's disease (PD) diagnosis by neurologists was only $65-75 \%$, which has not changed significantly in the last two decades [1].

Even in diseases that are hereditary with a clear genetic contribution, the clinical diagnosis remains difficult due to significant heterogeneity both in clinical features and genetic causes. This is evident in spinocerebellar ataxias (SCA) and hereditary spastic paraplegia (HSP). To date, 40 SCAs have been characterized, and 28 causal genes have been identified [2]. Genetically defined HSPs are assigned the symbol SPG (spastic gait) followed by a number. It is a highly heterogeneous group of disorders, with more than 80 genes or loci implicated [3]. There is significant overlap in clinical features of the two syndromes that 
makes diagnosis based on phenotype alone difficult. For example, patients with SCA1 may present initially with spastic paraplegia before development of cerebellar ataxia, and thus be misdiagnosed with HSP [4]. Conversely, patients with the HSP subtypes SPG4, SPG6, SPG31 and SPG37 may present with cerebellar atrophy [5].

These observations illustrate the shortcomings in the diagnosis of neurodegenerative disorders based on phenotype alone. The diagnostic criteria of these disorders are heavily reliant on clinical features, with little regard to the underlying etiology. Patients with similar clinical features due to different etiologies are often classified under the same diagnosis, resulting in a highly variable disease course and prognosis. The uncertainty in clinical diagnosis also renders recruitment of appropriate patients into research studies difficult, hindering the study of pathogenic mechanisms and the search for disease-modifying therapies.

In this review, we will discuss the role of genetic variants in hereditary and sporadic neurodegenerative disorders and the insights to etiology and pathogenic mechanisms afforded by advances in genetic sequencing and analysis. Possible mechanisms of pleitropy and future directions to help translate genetic findings to therapeutic interventions will be discussed.

\section{Genetics: insights into etiology}

While the etiology of common, sporadic neurodegenerative disorders is likely to be multifactorial and more difficult to dissect, hereditary conditions with Mendelian inheritance offer the opportunity to study pathogenic mechanism by identification of the causal genetic mutation and characterization of its effect on function. Early success in genetic linkage studies in the 1980's led to discovery of large numbers of genetic causes of monogenic diseases. It was discovered that patients diagnosed with one disorder based on phenotype often had many different genetic causes, as illustrated by the vast number of mutations associated with SCA and HSP. Using cell-based or animal-based studies, it became possible to define the pathogenic mechanism of a mutation, shedding light on cellular pathways involved in creating an abnormal phenotype, thus opening up therapeutic opportunity. Phenotype-genotype correlation also became possible, and classification of diseases such as SCA, HSP and Charcot-Marie Tooth disease (CMT) based on causative loci/genes has become widely accepted. Recognition of phenotype-genotype relationship also facilitates counseling on genetic testing and prognosis.

\section{Next generation sequencing}

One limiting factor hindering the more widespread application of genetic diagnosis was the method of DNA sequencing. Published by Frederick Sanger in 1977 [6], Sanger (also known as chain-termination) sequencing has been the most commonly used DNA sequencing method and is still considered to be the gold standard [7]. However, this method is time consuming and expensive, as evidenced by the tremendous amount of time (13 years) and money (USD $\$ 2.7$ billion) spent to complete the Human Genome Project $[8,9]$.

The arrival of next generation sequencing (NGS) platform in 2009 has resulted in a marked acceleration of the process of identifying genetic mutations. NGS offers the ability to simultaneously sequence large number of genomic regions. The whole genome can be sequenced (whole genome sequencing, or WGS); whereas when combined with techniques of targeted capture, any subset of the genome can be sequenced, an example being whole exome sequencing (WES) [10, 11]. Currently, using NGS technology, the whole human genome can be sequenced in 2 days at a cost of under USD $\$ 3000$, which continues to decline (Table 1).

Data generated by NGS is analyzed using various bioinformatics tools (examples in [12, 13]). In principle, genetic variants called from NGS data are filtered and prioritized following the general strategy as outlined in Fig. 1, with the goal to select for variants that are rare, nonsynonymous, compatible with the presumed inheritance pattern and predicted to be deleterious. Selected variants are then subjected to further validation by segregation analyses and pathogenicity studies (reviewed in [14]).

Before NGS, genetic mutations were identified mainly by positional cloning and linkage analysis which required large pedigrees with sufficient numbers of affected and unaffected individuals. Identification of mutations in patients with clinically and genetically heterogeneous disorders was limited by the large number of candidate genes and the associated high cost of Sanger sequencing. Moreover, in the clinical setting, diagnosis could not be established in patients with suspected underlying genetic disorders if they were not found to harbor any mutations in known causal genes. The advent of NGS changed the landscape of genetic diagnosis and offers a genomewide, unbiased approach which weighs all genes equally, allowing all identified variants to be simultaneously assessed. WES has been advocated as the standard of care in patients presenting with heterogeneous clinical phenotypes that could result from a wide variety of genetic causes $[15,16]$.

\section{Gene variants in rare Mendelian disorders}

Much of the early application and success of NGS is seen in rare Mendelian neurological disorders of high penetrance. In particular, WES, which captures all known coding exons where $85 \%$ of causal mutations are estimated to be located, has been utilized most widely. 


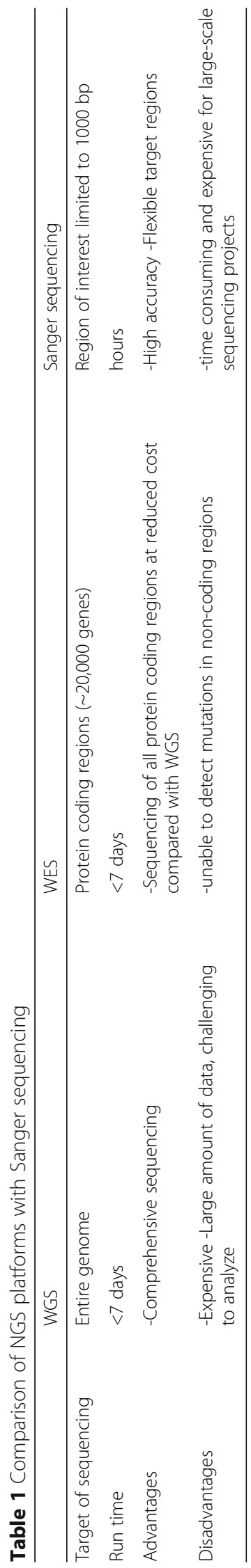




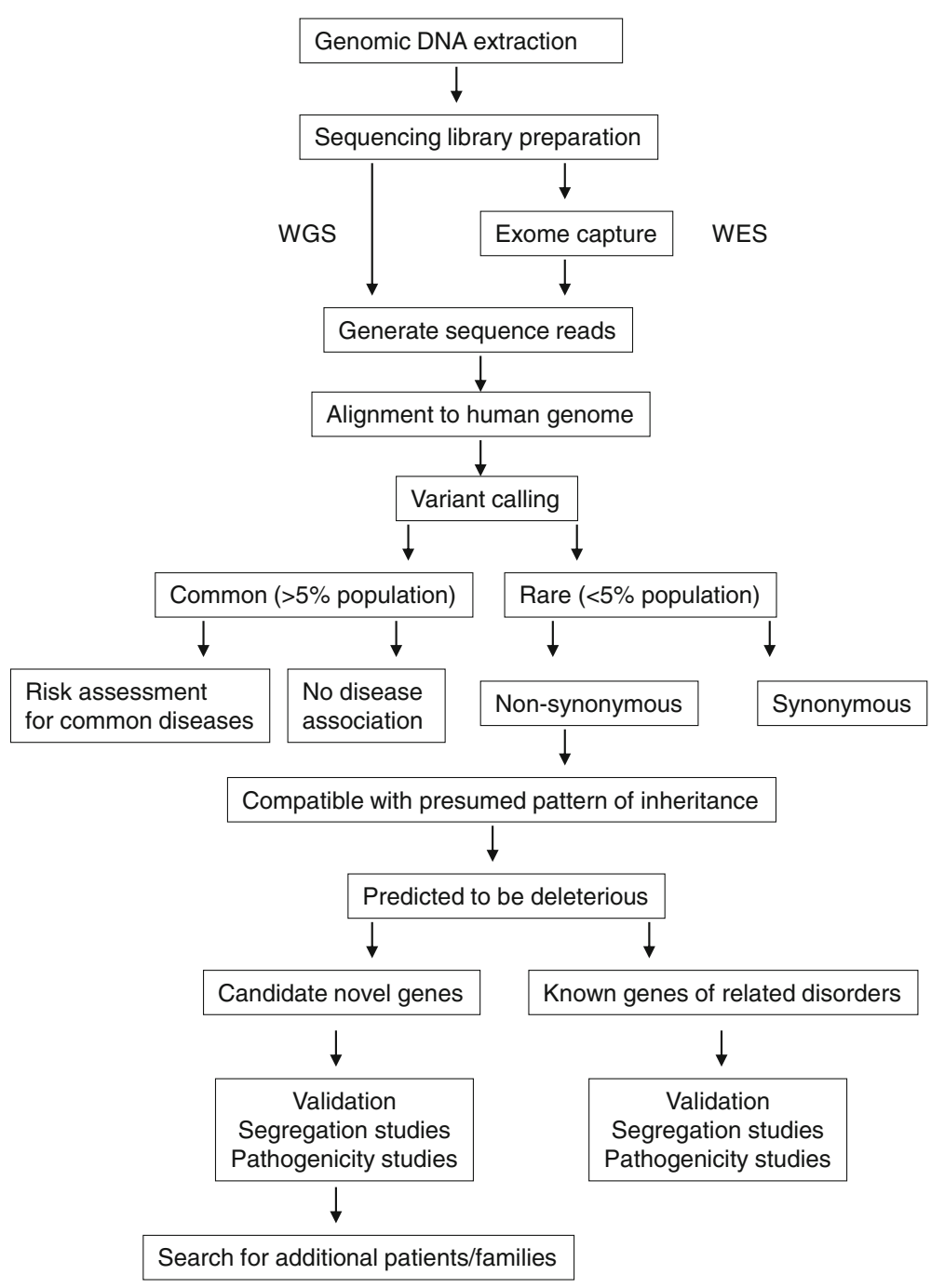

Fig. 1 Typical workflow of NGS and bioinformatic analysis. Genomic DNA is extracted and massive parallel sequencing on various NGS platforms is performed. For whole exome sequencing, exomes capture is performed, and the raw sequences are aligned to reference genome. Variant calling is performed and filtered by various softwares. Common variants can be further analyzed for risk assessment in common diseases. Non-synonymous and rare variants can be further scrutinized according to the presumed inheritance pattern and deleteriousness predictions. Candidate variants can be confirmed by validation, segregation analyses and pathogenicity studies. For novel variants, additional affected patients and families with the same variant can further strengthen the association of the variant with disease

The obvious advantage of WES in clinically and genetically heterogeneous disorders is the quick identification of mutations in known genes. When no known mutation can be found, WES offers the opportunity of identifying causal mutations in novel genes.

Using NGS, at least 36 novel genes have been identified which caused CMT (reviewed in [14]). Similarly, the list of causal genes in SCA has also expanded. For example, using WES combined with linkage analysis, mutations in TGM6 were identified in two Chinese families with SCA [17]. The importance of TGM6 in SCA in Chinese was confirmed when WES showed a third pedigree to have a novel mutation in the same gene [18]. Two studies using WES identified mutations in the voltage-gated potassium channel encoding gene KCND3 in families with autosomal dominant SCA, implicating reduced potassium channel function in the pathogenesis of SCA [19, 20]. Since 2012, at least 16 new genes have been identified to cause SCA (reviewed in [21]). WES was also used to identify PMCA4 as a novel gene in a Chinese family with HSP [22]. The effect of the mutation was studied using cell-based assays which demonstrated impaired intracellular calcium extrusion, implicating dysfunction of calcium homeostasis in the development of spastic paraplegia [23].

An important role of NGS lies in its ability to uncover novel genetic causes in patients who do not harbor any known causal mutations. For example, mutations in PMP22, GJB1, MFN2 and MPZ together account for about 
$86 \%$ of patients with CMT, with more than 50 other known genetic forms of CMT each accounting for less than 1 to $2 \%$ of patients [24]. If patients with additional associated neurological and non-neurological features are included, as many as $50 \%$ of patients with inherited peripheral neuropathy (IPN) are genetically undiagnosed [25]. Using WES in 50 families with molecularly undiagnosed IPN, $24 \%$ were found to have pathogenic or likely pathogenic variants in genes known to cause IPN, with an additional $22 \%$ harboring sequence variants of uncertain significance in IPN genes. In addition, $12 \%$ had variants in novel IPN candidate genes [26]. Another study showed that 21 of 110 index patients with IPN of unknown genetic etiology carried a sequence variant in a known CMT gene [27]. This finding was further extended in another study, which showed that 17 out of 37 (45\%) families with genetically undiagnosed CMT were found to have apparent causal mutations by WES, with 3 novel candidate genes identified [28]. Similarly, in HSP where there are more than 70 genetic subtypes described, NGS techniques have helped to identify the genetic diagnosis in 240 of 519 families [29]. In SCA, 44\% of French patients with autosomal dominant ataxia and $25 \%$ of Japanese familial ataxia patients remained genetically undiagnosed after SCA gene panel testing [30, 31]. Clearly, a notable proportion of patients with cerebellar ataxia have a genetic cause that remains to be identified, and NGS techniques will be invaluable in uncovering more causal genes.

\section{Gene variants in common and complex disorders}

Many neurodegenerative disorders that are common and predominantly sporadic also have familial forms. Examples include Parkinson's disease (PD), amyotrophic lateral sclerosis (ALS) and Alzheimer's disease (AD). Known genetic causes of familial forms only accounted for a small proportion of patients. Just as NGS is useful in the search for novel mutations in rare Mendelian disorders, it is also invaluable in uncovering genetic causes in unexplained familial forms of these common disorders. For example, about $10 \%$ of ALS cases are familial. The four genes well known to cause familial ALS, namely SOD1, TDP-43, FUS and VCP, together only account for $50 \%$ of familial cases (reviewed in [32]). Novel mutations in $S O D 1$ and $V C P$ were discovered through WES, as well as novel genes such as C9orf72, PFN1, UBQLN2. Similarly, about $5-10 \%$ of PD cases are familial, with 6 genes known to cause monogenic forms. LRRK2 mutations, the most common genetic cause of familial PD, only account for about $10 \%$ of familial PD cases [33]. WES identified novel genes that caused familial PD such as VPS35, EIF4G1 and DNAJC6 (reviewed in [34]).
Despite the success in uncovering novel genes in familial forms, the majority of patients with these disorders are sporadic with unknown etiology, probably involving a combination of genetic and environmental risk factors. Compared with Mendelian disorders, sporadic neurodegenerative disorders are common. Genetic contribution to these disorders has therefore been postulated to be through a combination of common genetic variants, each of which increases disease risk only slightly. Genome-wide association studies (GWAS) have been used to search for association between common genetic variants and a trait. In the field of PD, GWAS have identified 28 independent risk loci [35]. However, like most GWAS hits, each of these loci individually confers only modest risk. Heritability of PD has been estimated to be about 30\%, and risk loci identified by GWAS only accounted for one-tenth of this heritability [36]. Similarly, a recent GWAS estimated that single nucleotide polymorphisms (SNPs)-based heritability of ALS to be $8.5 \%$, with genome-wide significant loci only accounting for $0.2 \%$ of that heritability [37]. A genome-wide complex trait analysis estimated the heritability of all types of ALS to be $21 \%$ [38], and twin studies showed ALS heritability to be around $61 \%$ in sporadic cases [39]. Clearly, many more heritability factors remain to be identified which could be due to risk alleles that are too rare to detect using GWAS.

Studies utilizing targeted sequencing of known causal genes in sporadic cases have shown that genetic mutations in familial cases were also found in sporadic ones. For example, genetic mutations in familial ALS genes were found in $11 \%$ of sporadic patients (reviewed in [40]). Another study reported LRRK2 mutations in sporadic PD patients [33]. With the advent of WES, all exons can be sequenced simultaneously in large numbers of sporadic patients, thus not only enabling the search for genetic causes in sporadic cases, but also offers the opportunity to look for possibility of oligogenic inheritance in these diseases.

The concept of oligogenic inheritance in ALS was raised when some familial ALS (FALS) patients with a known causal mutation were found to harbor an additional mutation in another ALS risk gene. Using NGS techniques, a study showed that there was a significant enrichment of deleterious rare or novel alleles in ALS patients compared with controls, and that $60 \%$ of sporadic ALS (SALS) patients had rare or novel variants in 169 ALS genes [41]. With 40 candidate ALS genes, another study showed that $60.8 \%$ of SALS had at least one and $19.5 \%$ had two or more rare variants [42]. Moreover, it has been shown that increased risk variant burden is associated with earlier age of onset [43] and reduced survival [42] (Table 2). These studies showed that a significant proportion of sporadic 


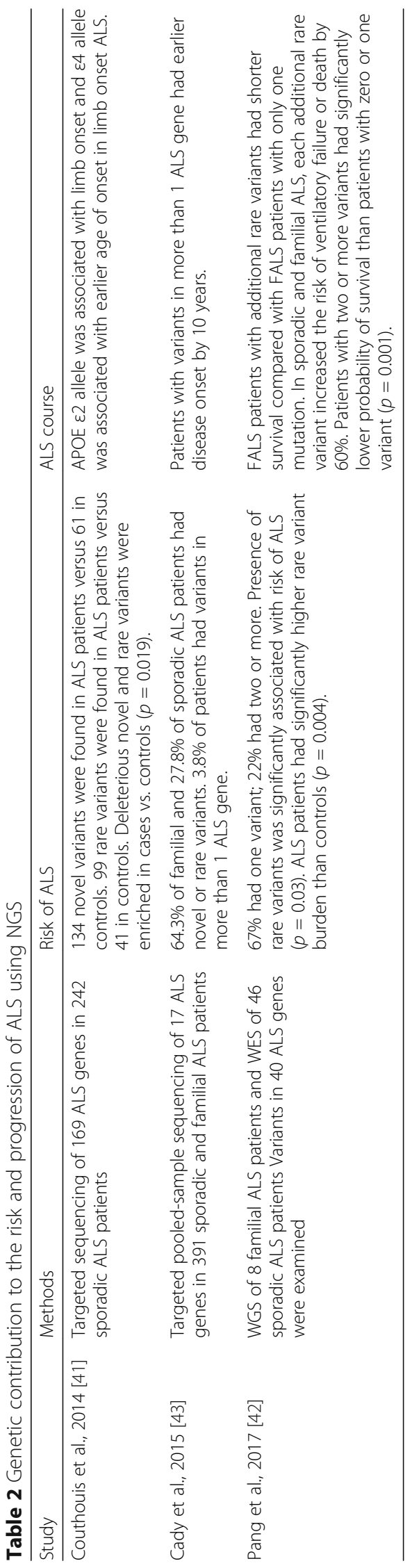


patients harbored rare variants in ALS risk genes, and help to identify the missing heritability of ALS. These observations are also supportive of an oligogenic influence on the development and progression of ALS.

Recently, a large-scale study using WES and involving more than 7000 PD patients showed that $30 \%$ of PD patients with a known primary genetic cause had additional rare variants in established PD-related genes compared with $17 \%$ in PD patients without known mutations and $15.6 \%$ in controls. Moreover, carriers of additional variants had earlier age of onset, suggesting an oligogenic inheritance in PD [44]. Undoubtedly, with the costs of NGS continuing to decrease, further studies will uncover more rare variants to be involved in the pathogenesis of complex neurodegenerative disorders, thereby elucidating cellular pathways that may be targets for intervention.

\section{Pleiotropy}

Just as clinically similar symptoms can be caused by a large number of genetic variants in various neurodegenerative disorders, a genetic locus can harbor variants that are associated with multiple, sometimes distinct, traits, giving rise to the concept of pleiotropy (reviewed in [45]). The mechanisms underlying pleiotropy is likely to be diverse, and understanding of these mechanisms will be crucial in our understanding of the genetic contribution and pathogenesis of neurodegenerative disorders (Table 3).

\section{Diverse phenotype due to mutations in the same gene}

Mutations in the same gene can often cause different phenotypes. For example, mutations in CACNA1A were described in SCA6, familial hemiplegic migraine type I and episodic ataxia type II (reviewed in [21]). Another gene, $K C N D 3$, previously known to cause cardiomyopathy, was also found to be implicated in autosomal dominant SCA $[19,20]$. Mutations in BSCL2 can cause different motor manifestations such as hereditary motor neuropathy, CMT2, SPG17 and has been described in a patient with rapidly progressive ALS-like phenotype [46]. VCP mutations are found in ALS, inclusion body myositis, HSP and CMT2 [47]. Interestingly, a TGM6 mutation which has been implicated in SCA was found to segregate with acute myeloid leukemia [48]. Possible explanations for pleiotropy observed are: (i) different mutations in the same gene can have different downstream effects leading to different phenotypes, (ii) mutations in the same gene can have different effects in different cell lines, as exemplified by TGM6 mutation in SCA and acute leukemia.

Different phenotype may also result from different variants in the same gene which confers different levels of pathogenicity. For example, while point mutations in the $\alpha$-synuclein encoding gene $S N C A$ are highly penetrant and cause young-onset PD, dosage mutations have more variable penetrance depending on copy number. In contrast, common variants of SNCA increase risk of PD only slightly [36].

\section{Diverse phenotype with same mutation}

Even within families with the same underlying causal mutation, there can be significant variability in phenotype in terms of symptoms, age of onset and disease course. For example, four patients from the same HSP pedigree with the same mutation in BSCL2 had wide variation in phenotype ranging from asymptomatic with only electrophysiological abnormality to a rapidly progressive ALS-like phenotype [46]. Another example is the wide phenotypic variability observed in a threegeneration Chinese pedigree with ALS due to the same SOD1 mutation with some patients having bulbar onset and rapid progression while others survived much longer [49]. These and other similar observations have led to the search for modifier genes to explain the clinical heterogeneity observed with the same causal mutation.

One example of a modifier gene is TMEM106b which has been found to have modifying effect on the risk of fronto-temporal lobar degeneration with accumulation of the TAR DNA binding protein 43 (FTLD-TDP). The two most common genetic causes of FTLD-TDP are mutations in progranulin gene $(G R N)$ and hexanucleotide repeat expansion in C9orf72. Patients carrying two copies of the minor allele of three TMEM106b SNPs were found to be under-represented in GRN mutation carriers with FTLD-TDP; in other words, homozygosity in the minor allele was protective (reviewed in [50]). Intriguingly, after the discovery of C9orf 72 repeat expansion as a genetic cause of FTLD as well as ALS with TDP-43 pathology, it was found that the minor allele in TMEM106b also protected against development of FTD, but not against ALS, in C9orf72 expansion carriers [51]. Conversely, ALS patients with C9orf72 repeat expansion and the protective TMEM106b alleles had better cognitive performance. While these observations confirm the modifying effect TMEM106B genotype has on the risk of developing FTD, they also raise the question of why there is a differential effect on the cortex and on the spinal cord, when the underlying mutation (C9orf72 repeat expansion) and the pathology (TDP-43 aggregation) are the same.

Another possible mechanism to explain pleiotropy resulting in diverse phenotypes with the same causal mutation is oligogenic inheritance. NSG has been very successful in this regard because it confers the advantage of detecting variants in all genes simultaneously. Again using ALS as an example, it was noted that significant heterogeneity within the same kindred existed in familial 


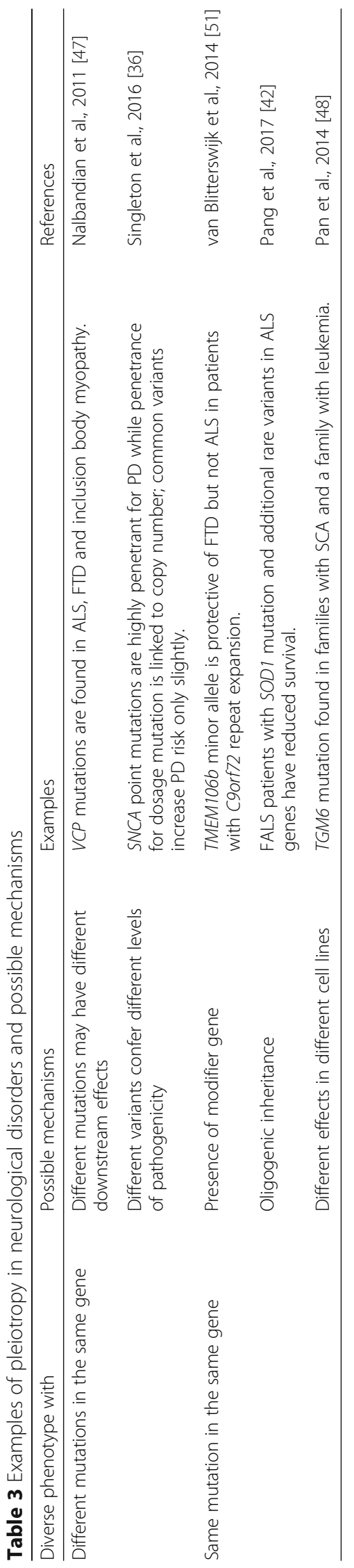


ALS with SOD1 mutation [49]. A recent study showed that within this pedigree, patients who had additional rare variants in 40 ALS genes had the most rapid disease progression and smallest chance of survival [42], raising the possibility that even with a highly penetrant causal mutation, the disease course is still modifiable by additional variants. This observation was further extended to sporadic cases, with patients harboring two or more rare variants having significantly lower survival than patients with zero or one rare variant. Another study showed that ALS patients with rare variants in more than one ALS gene had earlier age of onset [43]. These observations suggest that the development and progression of ALS likely involve a combined effect of multiple genetic variants, and clarification of pathways involving these genes will identify possible therapeutic targets for disease modification.

A recent study showed the possible oligogenic influence on PD, with about $30 \%$ of PD patients with a known causal mutation harboring additional variants [44]. Carriers of additional variants were found to have earlier age of onset. The most common causal mutation in this study was LRRK2 G2019S, and the most common additional variant in these patients occurred in ATP13A2. Thus, the presence or absence of additional variants in LRRK2 mutation carriers may account for the variable penetrance observed.

\section{Conclusion}

NGS has revolutionized the field of genetics in neurological disorders. The list of genes responsible for neurological dysfunction is rapidly expanding, helping to fill the gap of missing heritability in many neurodegenerative disorders. Identification of a novel gene leads to exploration of a new pathway in the pathogenesis of a disease, and genetic discovery is a starting point for elucidating pathogenic mechanisms. With the ever-reducing costs of NGS, the challenges ahead will be accurate analysis and interpretation of large amount of genetic data, and translating genetic data into pathogenic mechanisms and ultimately therapeutic opportunities. So far, WES has been most widely used, but obvious limitations include the inability to detect non-coding variants in intronic regions, large fragment copy number variations and chromosome derangement. Another challenge lies in the separation of benign from disease-causing variants. It has been estimated that in the genome of a single individual, about 10,000 to 11,000 nonsynonymous variants exist $[52,53]$. It has also been reported that up to $27 \%$ of allegedly disease-causing variants were later found to be common benign polymorphisms [54]. Future directions include better tools to differentiate pathogenic from benign variants at the bioinformatic and functional levels, collection of more human genome data of different ethnicities, collaborative effort to establish extensive public databases, and methods to study non-coding variants, functional genomics, and epigenetics.

The observation of pleiotropy suggests that common pathological mechanisms may underlie clinically diverse neurological syndromes. Through identification of new and sometimes common genes in different disorders, it has become clear that common pathways exist in the pathogenesis of neurodegeneration: aberrant ion channel function, mitochondrial dysfunction, defects in intracellular trafficking and axonal transport, abnormal protein aggregation and clearance. Much work still needs to be done to elucidate cellular targets for intervention. With the advent of NGS, genetic diagnosis is already replacing phenotype-oriented classification of neurological disorders, and individualized treatment based on genetic findings may one day be available to treat neurodegenerative diseases.

\section{Abbreviations \\ AD: Alzheimer's disease; ALS: Amyotrophic lateral sclerosis; CMT: Charcot- Marie Tooth; FALS: Familial ALS; FTLD-TDP: Fronto-temporal lobar degeneration with accumulation of the TAR DNA binding protein 43; GWAS: Genome-wide association study; HSP: Hereditary spastic paraplegia; IPN: Inherited peripheral neuropathy; NGS: Next generation sequencing; PD: Parkinson's disease; SALS: Sporadic ALS; SCA: Spinocerebellar ataxia; SNP: Single nucleotide polymorphism; SPG: Spastic gait; WES: Whole exome sequencing; WGS: Whole genome sequencing}

\section{Acknowledgements}

We would like to thank Liu Po Shan/Dr. Vincent Liu Endowment Fund for Motor Neurone Disease, the Henry G. Leong Professorship in Neurology, the Donation Fund for Neurology Research for their support.

\section{Funding}

This review was supported in part by Liu Po Shan/Dr. Vincent Liu Endowment Fund for Motor Neurone Disease.

Availability of data and materials

Not applicable.

\section{Authors' contributions}

SP: Forming the concept, drafting and revising the manuscript; All other authors: revising the manuscript. All authors read and approved the final manuscript.

Ethics approval and consent to participate

Not applicable.

\section{Consent for publication}

Not applicable.

\section{Competing interests}

The authors declare that they have no competing interests.

\section{Author details}

${ }^{1}$ Division of Neurology, Department of Medicine, Queen Mary Hospital, University of Hong Kong, Hong Kong, People's Republic of China. ${ }^{2}$ Centre for Genomic Sciences, University of Hong Kong, Hong Kong, People's Republic of China. ${ }^{3}$ Department of Medical Genetics, Zhongshan School of Medicine, Sun Yat-sen University, Guangzhou, People's Republic of China. ${ }^{4}$ Key Laboratory of Tropical Disease Control (SYSU), Ministry of Education, Guangzhou, People's Republic of China. 
Received: 30 June 2017 Accepted: 2 October 2017

Published online: 06 October 2017

\section{References}

1. Rajput AH, Rajput A. Accuracy of Parkinson disease diagnosis unchanged in 2 decades. Neurology. 2014;83:386-7.

2. Sun YM, Ly C, Wu ZY. Spinocerebellar ataxia: relationship between phenotype and genotype - a review. Clin Genet. 2016;90:305-14.

3. Klebe S, Stevanin G, Depienne C. Clinical and genetic heterogeneity in hereditary spastic paraplegias: from SPG1 to SPG72 and still counting. Rev Neurol (Paris). 2015;171:505-30.

4. Pedroso JL, de Souza PV, Pinto WB, Braga-Neto P, Albuquerque MV, SaraivaPereira ML, et al. SCA1 patients may present as hereditary spastic paraplegia and must be included in spastic-ataxias group. Parkinsonism Relat Disord. 2015;21:1243-6.

5. Finsterer J, Loscher W, Quasthoff S, Wanschitz J, Auer-Grumbach M, Stevanin G. Hereditary spastic paraplegias with autosomal dominant, recessive, X-linked, or maternal trait of inheritance. J Neurol Sci. 2012;318:1-18.

6. Sanger F, Nicklen S, Coulson AR. DNA sequencing with chain-terminating inhibitors. Proc Natl Acad Sci U S A. 1977:74:5463-7.

7. Fogel BL, Lee H, Strom SP, Deignan JL, Helson SF. Clinical exome sequencing in neurogenetic and neuropsychiatric disorders. Ann N Y Acad Sci. 2016;1366:49-60

8. Lander ES, Linton LM, Birren B, Nusbaum C, Zody MC, Baldwin J, et al. Initial sequencing and analysis of the human genome. Nature. 2001;409:860-921.

9. Collins FS, Morgan M, Patrinos A. The Human Genome Project: lessons from large-scale biology. Science. 2003;300:286-90.

10. Choi M, Scholl UI, Ju W, Liu T, Tikhonova IR, Zumbo P, et al. Genetic diagnosis by whole exome capture and massively parallel DNA sequencing. Proc Natl Acad Sci U S A. 2009;106:19096-101.

11. Gnirke A, Melnikov A, Maguire J, Rogov P, LeProust EM, Brockman W, et al. Solution hybrid selection with ultra-long oligonucleotides for massively parallel targeted sequencing. Nat Biotechnol. 2009;27:182-9.

12. Wang $\mathrm{K}$, Li M, Hakonarson $\mathrm{H}$. ANNOVAR: functional annotation of genetic variants from high-throughput sequencing data. Nucleic Acids Res. 2010;38:e164.

13. Li MX, Gui HS, Kwan JS, Bao SY, Sham PC. A comprehensive framework for prioritizing variants in exome sequencing studies of Mendelian diseases. Nucleic Acids Res. 2012;40:e53.

14. Liu YT, Lee YC, Soong BW. What we have learned from the next-generation sequencing: contributions to the genetic diagnoses and understanding of pathomechanisms of neurodegenerative diseases. J Neurogenet. 2015:29:109-12.

15. Miyatake S, Matsumoto M. Genetics: clinical exome sequencing in neurology practice. Nat Rev Neurol. 2014;10:676-8.

16. Gomez CM, Das S. Clinical exome sequencing: the new standard in genetic diagnosis. JAMA Neurol. 2014;71:1215-6.

17. Wang JL, Yang $X$, Xia K, Hu ZM, Weng L, Jin X, et al. TGM6 identified as a novel causative gene of spinocerebellar ataxias using exome sequencing. Brain. 2010;133:3510-8

18. Li M, Pang SY, Song Y, Kung MH, Ho SL, Sham PC. Whole exome sequencing identifies a novel mutation in the transglutaminase 6 gene for spinocerebellar ataxia in a Chinese family. Clin Genet. 2013;83:269-73.

19. Lee YC, Durr A, Majczenko K, Huang YH, Liu YC, Lien CC, et al. Mutation sin KCND3 cause spinocerebellar ataxia type 22. Ann Neurol. 2012;72:859-69.

20. Duarri A, Jezierska J, Fokkens M, Meijer M, Schelhaas HJ, den Dunnen WF, et al. Mutations in potassium channel kcnd3 cause spinocerebellar ataxia type 19. Ann Neurol. 2012;72:870-80.

21. Coutelier M, Stevanin G, Brice A. Genetic landscape remodelling in spinocerebellar ataxias: the influence of next-generation sequencing. J Neurol. 2015;262:2383-95.

22. Li M, Ho PW, Pang SY, Tse ZH, Kung MH, Sham PC, Ho SL. PMCA4 (ATP2B4) mutation in familial spastic paraplegia. PLoS One. 2014;9:e104790.

23. Ho PW, Pang SY, Li M, Tse ZH, Kung MH, Sham PC, et al. PMCA4 (ATP2B4) mutation in familial spastic paraplegia causes delay in intracellular calcium extrusion. Brain Behav. 2015;5:e00321

24. Hoyle JC, Isfort MC, Roggenbuck J, Arnold WD. The genetics of CharcotMarie-Tooth disease: current trends and future implications for diagnosis and management. Appl Clin Genet. 2015;8:235-43.

25. Murphy SM, Laura M, Fawcett K, Pandraud A, Liu YT, Davidson GL, et al. Charcot-Marie-Tooth disease: frequency of genetic subtypes and guidelines for genetic testing. J Neurol Neurosurg Psychiatry. 2012:83:706-10.
26. Hartley T, Wagner JD, Warman-Chardon J, Tétreault M, Brady L, Baker S, et al. Whole-exome sequencing is a valuable diagnostic tool for inherited peripheral neuropathies: outcomes from a cohort of 50 families. Clin Genet. 2017; https://doi.org/10.1111/cge.13101.

27. Drew AP, Zhu D, Kidambi A, Ly C, Tey S, Brewer MH, et al. Improved inherited peripheral neuropathy genetic diagnosis by whole-exome sequencing. Mol Genet Genomic Med. 2015;3:143-54.

28. Gonzaga-Jauregui C, Harel T, Gambin T, Kousi M, Griffin LB, Francescatto L, et al. Exome sequence analysis suggests genetic burden contributes to phenotypic variability and complex neuropathy. Cell Rep. 2015;12:1169-83.

29. Schüle R, Wiethoff S, Martus P, Karle KN, Otto S, Klebe S, et al. Hereditary spastic paraplegia: clinicogenetic lessons from 608 patients. Ann Neurol. 2016;79:646-58.

30. Degardin A, Dobbelaere D, Vuillaume I, Defoort-Dhellemmes S, Hurtevent JF, Sablonnière B, et al. Spinocerebellar ataxia: a rational approach to aetiological diagnosis. Cerebellum. 2012;11:289-99.

31. Sakakibara R, Tateno F, Kishi M, Tsuyusaki Y, Aiba Y, Terada H, et al. Genetic screening for spinocerebellar ataxia genes in a Japanese single-hospital cohort. J Mov Disord. 2017; 10.14802/jmd.17011.

32. Jiang T, Tan MS, Tan L, Yu JT. Application of next-generation sequencing technologies in Neurology. Ann Transl Med. 2014:2:125.

33. Berg D, Schweitzer $K$, Leitner $P$, Zimprich A, Lichtner $P$, Belcredi $P$, et al. Type and frequency of mutations in the LRRK2 gene in familial and sporadic Parkinson's disease. Brain. 2005;128:3000-11.

34. Kalinderi K, Bostantjopoulou S, Fidani L. The genetic background of Parkinson's disease: current progress and future prospects. Acta Neurol Scand. 2016;134:314-26.

35. Nalls M, Pankratz N, Lill CM, Do CB, Hernandez DG, Saad M, et al. Largescale meta-analysis of genome-wide association data identifies six new risk loci for Parkinson's disease. Nat Genet. 2014:46:989-93.

36. Singleton A, Hardy J. The evolution of genetics: Alzheimer's and Parkinson's diseases. Neuron. 2016:90:1154-63.

37. van Rheenen W, Shatunov A, Dekker AM, McLaughlin RL, Diekstra FP, Pulit SL, et al. Genome-wide association analyses identify new risk variants and the genetic architecture of amyotrophic lateral sclerosis. Nat Genet. 2016:48:1043-8.

38. Keller MF, Ferrucci L, Singleton AB, Tienari PJ, Laaksovirta $H$, Restagno G, et al. Genome-wide analysis of the heritability of amyotrophic lateral sclerosis. JAMA Neurol. 2014;71:1123-4.

39. Al-Chalabi A, Fang F, Hanby MF, Leigh PN, Shaw CE, Ye W, Rijsdijk F. An estimate of amyotrophic lateral sclerosis heritability using twin data. J Neurol Neurosurg Psychiatry. 2010;81:1324-6.

40. Renton AE, Chio A, Traynor BJ. State of play in amyotrophic lateral sclerosis genetics. Nat Neurosci. 2014;17:17-23.

41. Couthouis J, Raphael AR, Daneshjou R, Gitler AD. Targeted exon capture and sequencing in sporadic amyotrophic lateral sclerosis. PLoS Genet. 2014; 10:e1004704.

42. Pang SY, Hsu JS, Teo KC, Li Y, MHW K, KSE C, et al. Burden of rare variants in ALS genes influences survival in familial and sporadic ALS. Neurobiol Aging. 2017; https://doi.org/10.1016/j.neurobiolaging.20176.06.007.

43. Cady J, Allred P, Bali T, Pestronk A, Goate A, Miller TM, et al. ALS onset is influenced by the burden of rare variants in known ALS genes. Ann Neurol. 2015;77:100-13.

44. Lubbe SJ, Escott-Price V, Gibbs JR, Nalls MA, Bras J, Price TR, et al. Additional rare variant analysis in Parkinson's disease cases with and without known pathogenic mutations: evidence for oligogenic inheritance. Hum Mol Genet. 2016:25:5483-9.

45. Solovieff N, Cotsapas C, Lee PH, Purcell SM, Smoller JW. Pleiotropy in complex traits: challenges and strategies. Nat Rev Genet. 2013;14:483-95.

46. Musacchio T, Zaum AK, Uceyler N, Sommer C, Pfeifroth N, Reiners K, et al. ALS and MMN mimis in patients with BSCL2 mutations: the expanding clinical spectrum of SPG17 hereditary spastic paraplegia. J Neurol. 2016; https://doi.org/10.1007/s00415-016-8301-2.

47. Nalbandian A, Donkervoort S, Dec E, et al. The multiple faces of valosincontaining protein associated diseases: inclusion body myopathy with Paget's disease of bone, frontotemporal dementia, and amyotrophic lateral sclerosis. J Mol Neurosci. 2011;45:522-31.

48. Pan LL, Huang YM, Wang M, Zhuang XE, Luo DF, Guo SC, et al. Positional cloning and next-generation sequencing identified a TGM6 mutation in a large Chinese pedigree with acute myeloid leukemia. Eur J Hum Genet 2014; https://doi.org/10.1038/ejhg.2014.67. 
49. Fong GCY, Kwok KHH, Song YQ, Cheng TS, Ho PW, Chu AC, et al. Clinical phenotypes of a large Chinese multigenerational kindred with autosomal dominant familial ALS due to lle149Thr SOD1 gene mutation. Amyotroph Lateral Scler. 2006;7:142-9.

50. Nicholson AM, Rademakers R. What we know about TMEM106B in neurodegeneration. Acta Neruopathol. 2016;132:639-51.

51. van Blitterswijk M, Mullen B, Nicholson AM, Bieniek KF, Heckman MG, Baker MC, et al. TMEM106B protects C9ORF72 expansion carriers against frontotemporal dementia. Acta Neuropathol. 2014;127:397-406.

52. Wheeler DA, Srinivasan M, Egholm M, Shen Y, Chen L, McGuire A, et al. The complete genome of an individual by massively parallel DNA sequencing. Nature. 2008;452:872-6.

53. Ng PC, Levy S, Huang J, Stockwell TB, Walenz BP, Li K, et al. Genetic variation in an individual human exome. PLoS Genet. 2008;4:e1000160.

54. Xue Y, Chen Y, Ayub Q, Huang N, Ball EV, Mort M, et al. Deleterious- and disease-allele prevalence in healthy individuals: insights from current predictions, mutation databases, and population-scale resequencing. Am J Hum Genet. 2012;91:1022-32

Submit your next manuscript to BioMed Central and we will help you at every step:

- We accept pre-submission inquiries

- Our selector tool helps you to find the most relevant journal

- We provide round the clock customer support

- Convenient online submission

- Thorough peer review

- Inclusion in PubMed and all major indexing services

- Maximum visibility for your research

Submit your manuscript at www.biomedcentral.com/submit
Biomed Central 